\title{
Haptic Aided Design: a Case Study
}

\author{
Jian Zhang, Shahram Payandeh and John Dill \\ Experimental Robotics and Graphics Laboratories \\ School of Engineering Science \\ Simon Fraser University \\ Burnaby, BC V5A 1S6, Canada \\ \{jzhanga|shahram|dill\}@cs.sfu.ca
}

\begin{abstract}
This paper presents a preliminary investigation of the effectiveness of haptic feedback for design processes involving mechanisms used by a human operator in interacting with an environment. Our example is a case study of the design of an amniocentesis device, which represents a relatively simple and yet meaningful application. We simulated the mechanism, as well as its intended working environment, represented by layered mass-spring surface meshes. The user feels force feedback when manipulating the simulated device via a haptic interface. The user can also interactively update the design by modifying the values of the parameters that describe the mechanism. To investigate the effectiveness of haptic rendering in this design problem, an informal preliminary user study was conducted, where each subject explored a discretized design space of the mechanism. The results of the user study suggest that haptic rendering is effective in expediting the design process for some applications.
\end{abstract}

\section{Introduction}

Computer Aided Design (CAD) is a useful tool for facilitating design processes. A designer can specify geometry and other properties of mechanisms using a CAD system, piece them together, specify relationships between parts, simulate movements of the mechanism, and export the result to files that manufacturers can use to build the mechanism. If a human operator is needed to manipulate the mechanism, it is important to determine if the design is ergonomically suitable for such interaction. Since most current CAD systems do not provide this feature, physical prototypes have to be built for ergonomic evaluation.

With the emergence of force feedback devices, utilization of haptic force feedback and haptic rendering in design process may become a useful feature. An operator can interact with the mechanism, and feel the forces involved. Force feedback gives the operator an appreciation of the way the mechanism interacts with the intended environment in a much more direct and intuitive way. Since in this approach force feedback is essential in the design process, we call it "Haptic Aided Design" or HAD.

Some previous research has made contributions toward incorporating force feedback into traditional CAD systems. For example, [1] describes a haptic interface coupled with CAD software, allowing the operator to both see and feel geometrical shapes and dynamics forces. In the work described by [2], the author formulated inverse kinematics and inverse dynamics equations involved in simulating open chain mechanisms and single closed chain mechanisms. [3] describes a formulation for force calculation in a virtual mechanism manipulation system to simulate the mechanics of two-finger grasping. [4] describes a virtual prototyping system with the capability of simulating two-finger grasping. Collision detection, force feedback, and a graphical representation of the operator's arm are also parts of this system.

[5] describes a system that integrates a full-body simulation environment called JACK ${ }^{\mathrm{TM}}$ and a Rutgers Master II four-finger haptic feedback device. In this system, the user uses verbal commands to control the body of a virtual human agent, while the hand movements of the virtual agent follows the user's hand movement via a haptic device, i.e. the Rutgers Master II. The user can control the virtual agent to grasp a tool on a workbench in the virtual environment, and command the virtual human agent to walk to another workbench and drop the tool there.

The above systems shed light on viable approaches to mechanism simulations with force feedback. However, whether or not haptic feedback is effective in those systems was not considered, which is an important question that needs to be answered. Our goal in this paper is to study whether or not haptic rendering helps expedite the mechanism design process. To achieve this goal, we developed a functional platform where a simple and yet practical mechanism is simulated with force feedback, and can be modified interactively. An informal user study was conducted to study the effectiveness of haptic feedback in our design environment.

Our approach in this paper is a case study. Our HAD environment takes the form of designing a mechanism for assisting with amniocentesis. Amniocentesis is a delicate procedure during which a needle is inserted through 
layers of tissue in the abdominal area of the candidate until it penetrates the amniotic sac. Then amniotic fluid is extracted for further examination. If the procedure is performed poorly, the fetus might be damaged. Traditionally this task is accomplished manually and thus may be subject to various uncertainties arising from operator strength and skills, and movement of the fetus. Hence, a mechanism that helps perform amniocentesis in a more reliable way is desirable.

In our proposed HAD environment, the designer can manipulate the mechanism and insert the attached needle through a multi-layered mesh structure used to model the tissue layers and feel the interaction forces created as a function of the movements of the mechanism. The designer can fine-tune important design parameters to achieve desired force characteristics of the mechanism that gives the best operator performance. In our design environment, this is measured by the amount of overshoot into the amniotic sac.

The rest of this paper is structured as follows. The platform is overviewed in section 2; the multi-layer tissue model, mechanism simulation, and collision detection are described in sections 3, 4, and 5, respectively. The informal preliminary user study conducted to test the effectiveness of haptic feedback in our HAD platform is described in section 6. Section 7 is conclusions and discussions.

\section{Overview of the HAD platform}

Figure 1 shows the user interface of our HAD environment

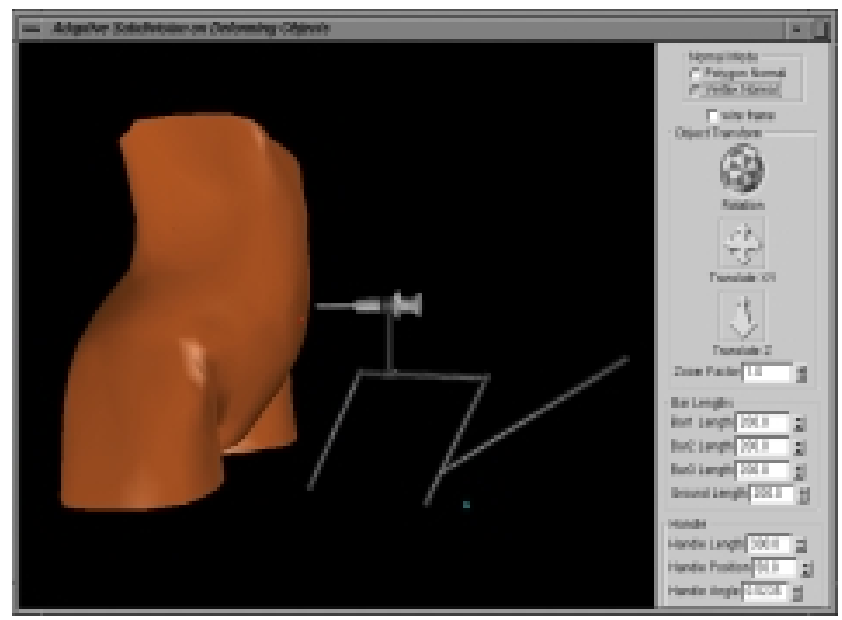

Figure 1: User interface of our HAD platform

The mechanism is represented by a four-bar linkage, with links $1,2,3$ and 4 corresponding to $l_{1}, l_{2}, l_{3}$ and $l_{4}$ in Figure 2. A needle is attached to link 2. Attached to link 3 is a representation of a handle. The tip of the Phantom stylus is represented as a small sphere in the virtual environment. On the left of Figure 1 is a three-layer human body model, representing the skin, muscle, and the amniotic sac layers. (The user only sees the outer skin layer). The skin and amniotic sac layers are represented as mass-spring meshes. The second layer is represented as a force field between the first and the third.

As the small sphere makes contact with a link or handle in the scene, the user feels its presence and can recognize the cylindrical shapes representing the mechanism links when sliding the Phantom tip on the surface of the links and the handle.

When the user is in contact with a link or the handle and presses the button on the Phantom stylus, the stylus is attached to the link or handle at the point of contact. As a result, movements of the user's hand connected to the link becomes an input to the mechanism. The user can also feel constraint forces when trying to move in directions restricted by the kinematic configuration of the mechanism. The three tissue layers generate forces as the needle tip makes contact with and penetrates them. These forces are then mapped to the contact point coordinates and then to the haptic device.

Figure 2 shows the mechanism parameters. The designer can interactively modify the kinematic configuration of the mechanism by changing parameter values via input controls shown on the right side of Figure 1. By changing the parameter values, it is possible to identify an optimal configuration of the mechanism that minimizes the overshoot into the amniotic sac, while remaining within the range of comfortable operation for the user.

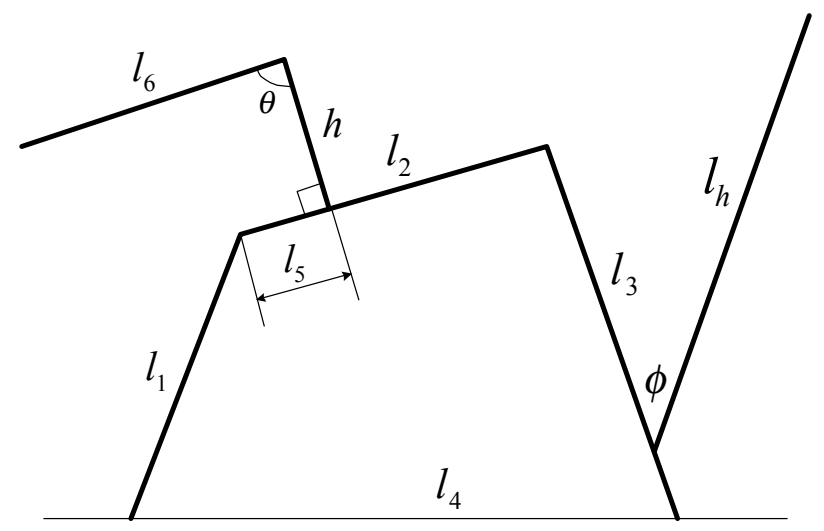

Figure 2: Parameters describing the mechanism.

\section{The multi-layer tissue model}

The total force exerted on the needle tip from our three-layer tissue model is the summation of the forces from individual layers:

$$
F=F_{l 1}+F_{l 2}+F_{l 3} .
$$


Force $F$ must be mapped to the location on the handle where the user interacts with the mechanism, before being sent to the Phantom haptic device for force display. This mapping is described in the next section. We now describe how the force from each layer is calculated.

At the instant the needle tip makes contact with the first layer, the triangle on the surface mesh in contact with the needle is identified. Neighborhood triangles of the contacted triangle are subdivided based on the local subdivision schemes proposed in [7] to provide the user with greater deformation detail in the local area. A new contact triangle in the subdivided area is then identified. The new contact triangle then follows the user's hand movement, and its neighborhood is thus deformed due to extension or compression of the springs. The force that is exerted on the needle tip due to first layer mesh deformation is

$$
F_{l 1}=F_{v 1}+F_{v 2}+F_{v 3},
$$

where $F_{l 1}$ is the force vector created due to the deformation of the first layer. $F_{v i}$ is the force vector to which the $i$ th vertex of the contact triangle is subject, as illustrated in Figure 3.
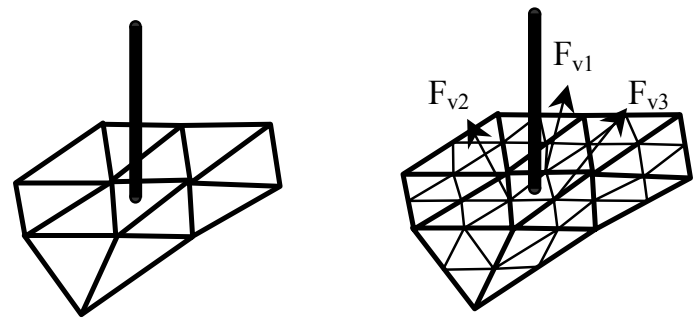

Figure 3: Local surface subdivision and reaction force calculation when probe makes contact with the skin layer.

If the magnitude of $F_{l 1}$ exceeds a preset threshold, the first layer is penetrated and the needle tip enters the second layer. The contacted triangle no longer follows the user's hand movement and the mesh representing the first layer relaxes to its original shape. After penetration, the friction force from the first layer is approximated by a small resistive force of a constant magnitude in the direction opposite to the movement of the needle tip:

$$
F_{l 1}=-C_{1} \frac{v}{|v|},
$$

where $C_{1}$ is a positive constant and $v$ is the velocity vector of the movement of the needle tip.

The second layer corresponds to the space between meshes representing the first and third layers. The user feels the presence of the second layer through its distinct force properties. For this layer we want to approximate coulomb friction forces. The coulomb friction force model of a needle inserted into tissue is different than that of one rigid object sliding on the surface of another rigid object. We approximate the static friction force when the tissue is deforming with a linear spring, and the dynamic friction force when the needle slides relative to the tissue with a damping force. When the needle tip stops moving, the position $\mathrm{P}_{\mathrm{s}}$ where it stops is recorded and a linear spring $K_{l 2}$ is attached between $\mathrm{P}_{\mathrm{s}}$ and the needle tip. Successive identical stylus coordinates returned by the Phantom determine where motion has stopped. The force exerted on the needle tip is then described by

$$
F_{l 2}=K_{l 2} R_{s} \text {, }
$$

where $F_{l 2}$ is the force exerted on the needle tip when it is in the second layer, $K_{l 2}$ is the spring constant and $R_{s}$ is the vector from the current needle tip $T$ to the recorded stop position $P_{s}$.

If the magnitude of $F_{l 2}$ exceeds a preset threshold, we switch from the "stick" stage of the Coulomb force model to the "slip" stage. The spring is removed and the friction force between the needle and the tissue is approximated by

$$
F_{l 2}=-D_{l 2} V_{T}
$$

where $D_{l 2}$ is a preset damping coefficient and $V_{T}$ is the velocity of the needle tip. At this point $F_{l 2}$ is a damping force from the second layer that is proportional to the velocity of the needle tip.

As the user pushes the needle further into the layered tissue model, the needle tip will make contact with the third layer, i.e. the representation of the amniotic sac. Local subdivision, deformation and force calculations for this layer are similar to those for the first layer as described in [7]. The force exerted on the needle tip from the third layer is calculated as the sum of the forces that the three vertices of the contacted triangle are subject to, similar to what we did for the first layer:

$$
F_{l 3}=F_{v 1}+F_{v 2}+F_{v 3} \text {. }
$$

If the magnitude of $F_{l 3}$ is beyond a specified threshold, the third layer ruptures and returns to its original shape, and the needle tip enters the amniotic sac. After penetration, the third layer only contributes a small resistive force of a constant magnitude in the direction opposite to the movement of the needle tip to approximate the friction force:

$$
F_{l 3}=-C_{3} \frac{v}{|v|} \text {. }
$$


In our study, the position where the user makes contact with the third layer is recorded. The user tries to stop as soon as possible after penetrating the third layer. Once the needle tip stops, the overshoot is reported to the user as the distance $d$ illustrated in Figure 4. For our study, a smaller overshoot signifies a better performance.
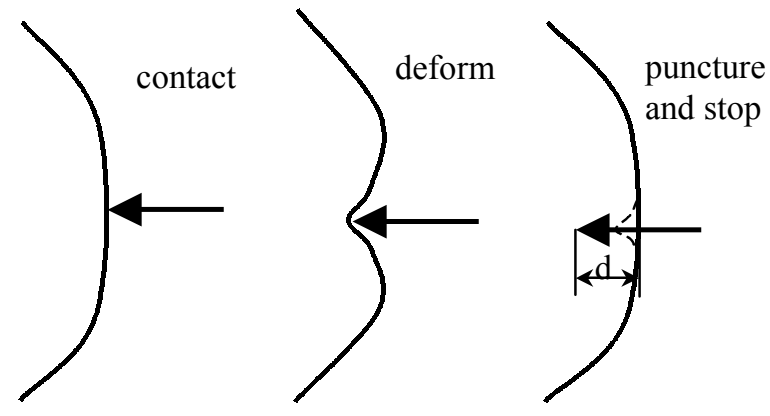

Figure 4: Calculation of overshoot d.

\section{Mechanism simulation}

The mechanism is a four-bar linkage (Figure 2). For this study we have excluded consideration of other types of possible mechanisms such has five-bar, six-bar or other higher mobility mechanisms. For our model, a needle is attached to link 2 and a handle is attached to link 3 . The links and handle are modeled with cylinders. Link and handle lengths are adjustable. The handle also has parameters for its position and the angle relative to the third link, which are also adjustable. The user grasps the handle and moves the mechanism to penetrate the threelayer model.

\subsection{Interactive kinematics and constraint force calculation}

In this section we show how the new positions and orientations of the links and the needle are determined when the user makes contact with the handle and moves the mechanism. Figure 5 shows details of link 3 and the handle; the user grasps the handle at point $G$ and moves the linkage. The user controls the position of the contact point by moving the Phantom stylus. In general, the mechanism follows the user's movement, but we do not want the user's hand to slide on the handle and we wish to constrain the motion to lie in the plane of the linkage. In Figure 5, the solid lines represent the orientations of link 3 and the handle at time $t_{0}$, and dashed lines represent their orientations at $t_{1}$. The user tries to move the Phantom end point from point $G$ to $T$. At time $t_{0}$ we know the coordinates of $\mathrm{O}, \mathrm{B}, \mathrm{C}$, and $\mathrm{D}$ and we wish to find the orientations for link 3 and the handle at time $t_{1}$.

Link 3 and the handle form a rigid body. When the user moves from $G$ to $T$, we rotate this rigid body by the corresponding angle so that rotation of the linkage follows the user's movement. Since the coordinates of G, O and T are known, angle $\angle G O T$ can be calculated. The new orientation of link $3, \overrightarrow{O B^{\prime}}$, is then obtained by rotating $\overrightarrow{O B}$ counterclockwise by $\angle G O T$. The position of $\mathrm{D}^{\prime}$ is located by the length of $\overrightarrow{O D}$ along $\overrightarrow{O B^{\prime}}$. Orientation of the handle $\overrightarrow{D^{\prime} C^{\prime}}$ is then obtained by rotating $\overrightarrow{B^{\prime} D^{\prime}}$ clockwise by angle $\angle B D G$.

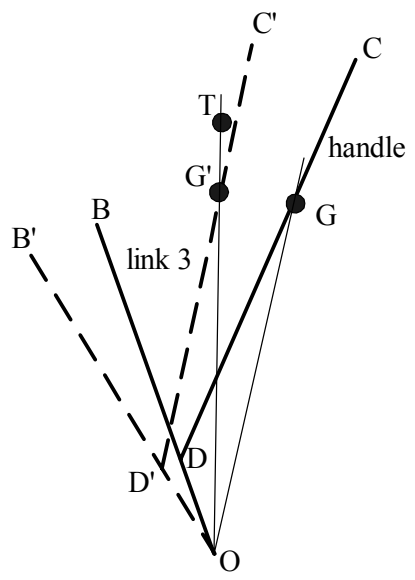

Figure 5: Determining the orientation of link 3 as the handle is being moved.

So far, we know how to orient the link and handle. Since the user is constrained not to slide on the handle, we now need to calculate the user's new contact position on the handle and the constraining forces (needed to "glue" the user's hand to that position). In Figure 5, G' can be located along vector $\overrightarrow{O T}$ with $\left|\overrightarrow{O G^{\prime}}\right|=|\overrightarrow{O G}|$ satisfied. The constraint force $F_{c}$ is calculated as:

$$
F_{c}=K_{c} \overrightarrow{T G^{\prime}}
$$

Where $K_{c}$ is a positive constant.

We can now determine the positions and orientations of link 1 and link 2. Figure 6 illustrates the needed angles, which can be calculated using basic trigonometry given $\alpha_{1}$ and link lengths.

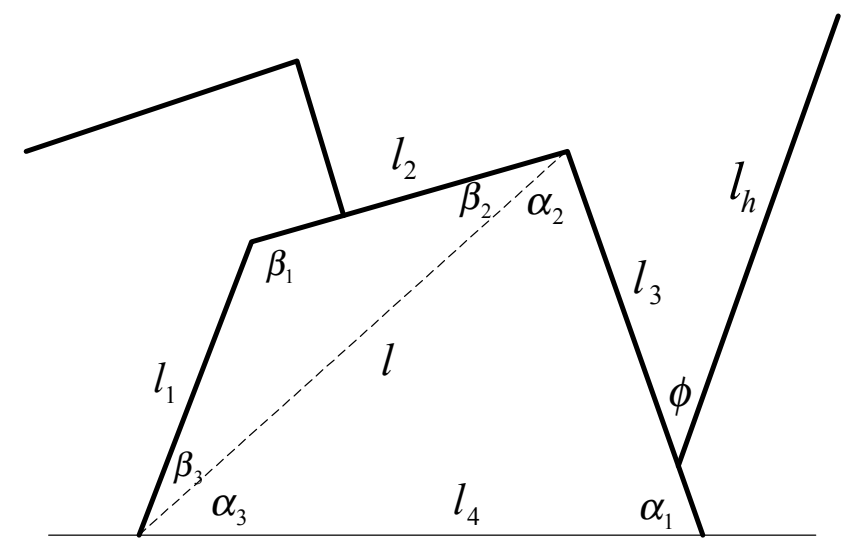


Figure 6: schematic of the four-bar linkage.

The final stop is to determine the new position of the needle tip, which is used to test for collision with the tissue model.

\section{Collision detection between needle and the tissue model}

In our proposed Amniocentesis simulation, collisions between needle tip and mesh layers need to be detected. In our polygon mesh data structure, neighbor elements of any given element are readily obtained. The basic idea of our collision detection algorithm involves two steps. First, we find the mesh vertex closest to the needle tip. Second, we check the triangles in the neighborhood of this vertex for collisions. The first step is illustrated in Figure 7.

Figure 7 illustrates a one-dimensional mesh of connected line segments. Suppose that at time $t_{0}$, the closest vertex is $B$, labeled with a small triangle. At $t_{1}$, the needle tip has moved to P'. We first check the neighbor vertices of $\mathrm{B}$, namely $\mathrm{A}$ and $\mathrm{C}$, and find the shortest distance among $\mathrm{AP}$ ', $\mathrm{BP}$ ' and $\mathrm{CP}$ '. In this case we find $\mathrm{CP}^{\prime}$ is the shortest, so $\mathrm{C}$ is labeled as the temporary closest vertex. We then repeat this process on $\mathrm{C}$ and find $\mathrm{D}$ is closer to $\mathrm{P}$ ' than $\mathrm{C}$ is, so $\mathrm{D}$ is now labeled as the closest vertex. Repeating the same process on $\mathrm{D}$ reveals that $\mathrm{D}$ is still the closest to $\mathrm{P}$ ' among $\mathrm{C}, \mathrm{D}$ and $\mathrm{E}$. This then suggests that $D$ is indeed the closest vertex at $t_{1}$. There are some cases where this is not the case; we discuss this in the Appendix. Fortunately the geometry of the tissue model we used does not impose such difficulties on our collision detection algorithm.
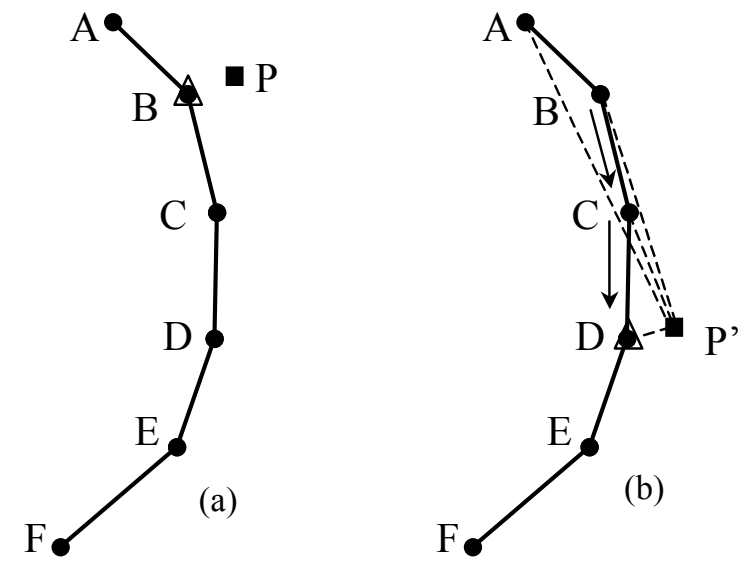

Figure 7: The first step of collision detection: find the closest vertex. $P$ and $P^{\prime}$ are needle tip positions; the small triangles mark the closest vertex to $P$ and $P$ '. (a) $t=t_{0}$. (b) $t=t_{1}$.

We now continue to the second step of our collision detection algorithm. The closest point provides a hint to where a collision might occur. We test to see if the line segment defined by the last and current position of needle tip intersects one of the triangles in the neighborhood of the closest vertex. If no intersected triangle is found, there has been no collision.

Two meshes of our tissue model are the skin layer and the amniotic sac layer. Our implementation maintains a closest vertex on each of them and checks for collisions between needle tip and triangles in the neighborhood of each of the closest vertices.

If the user's movement between two consecutive model updates is small compared to the size of the mesh, our collision detection algorithm takes little time because only the local area of the mesh is involved in the algorithm. Another advantage of our algorithm is that no additional computation is needed to update supporting data structures, such as the bounding volumes in some collision detection algorithms.

\section{An informal user study}

In this section we present the results of a preliminary user study of the proposed HAD environment. The main question that we ask is: Does our HAD environment suggest that haptics feedback is an effective tool in designing mechanisms a user manipulates and which interact with an environment? To investigate this question, we conducted an informal user study.

\subsection{Experiment Design}

The idea of the experiment is to use haptics to find an optimum (or at least a "good") set of design parameters for the device under study. We used overshoot as the criterion for determining optimality, which is illustrated in Figure 4. The stopping position is defined as several successive Phantom tip positions with identical coordinates. A good mechanism design is one that tends to generate a small overshoot for the average user.

The objective is to determine if we can find optimum mechanism configurations in easier and more intuitive ways with the help of haptic feedback. However, this approach is impractical since it would take a user too long to explore this high dimensional parameter space. Thus, we asked the subjects to try out only a small number of configurations of the mechanism.

The mechanism is described by the seven parameters illustrated in Figure 8.

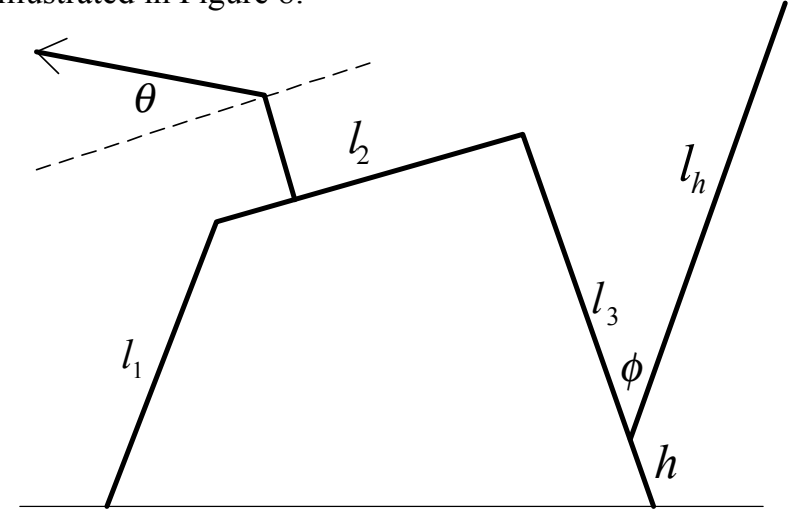


Figure 8: Parameters that define the mechanism.

$l_{1}, l_{2}$ and $l_{3}$ are lengths of links; $\theta$ is the angle of the needle with respect to any straight line parallel to the second link; $\phi$ is the angle of the handle with respect to the third link; $h$ is the position of the handle relative to the third link; $l_{h}$ is the length of the handle.

We chose discrete values for each parameter and combined them to form a finite set of mechanism configurations. These discrete values were chosen such that each configuration was significantly different from others, so that each portion of the design space has an entry in our set of configurations. For the lengths of the three bars that form the basic structure of a four-bar linkage, we identify three representative cases (Figure 9). We chose two values of $l_{h}$, three values of $\phi$ and two values of $h$ (Table 1$)$.
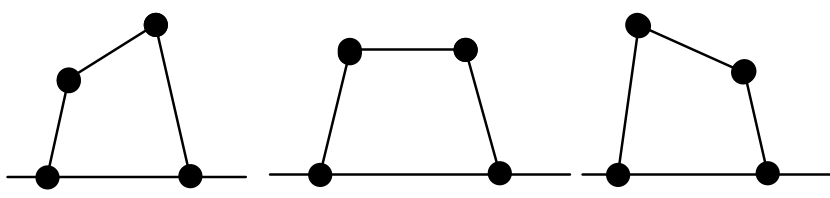

Figure 9: Three representative configurations for fourbar linkage.

Besides representing typical mechanism configurations, the parameter values were chosen such that: a) the workspace and force magnitude were comfortable for a human operator, and b) the workspace was not too large to be out of the range that the Phantom arm could reach. These requirements were met by a pilot study with two graduate students.

Table 1: Values of mechanism parameters used in user study.

\begin{tabular}{|c|c|c|c|c|}
\hline Parameters & $\begin{array}{c}l_{1}, l_{2}, l_{3} \\
(\mathrm{~mm}) \text { and } \theta \\
(\mathrm{rad})\end{array}$ & $\begin{array}{c}l_{h} \\
(\mathrm{~mm})\end{array}$ & $\phi(\mathrm{rad})$ & $\begin{array}{c}h \\
(\mathrm{~mm})\end{array}$ \\
\hline \multirow{5}{*}{ Values } & 80,150, & & \multirow{2}{*}{$0, \frac{\pi}{2}}$, & \\
& $120,-0.31$ & 100,100, & 140, & \multirow{2}{*}{0,80} \\
& $100,-0.17$ & 180 & $\frac{5 \pi}{6}$ & \\
\cline { 2 - 3 } & $\begin{array}{c}120,150, \\
80,0.31\end{array}$ & & & \\
\hline
\end{tabular}

At the beginning of the experiment, each subject was given a short introduction to the interface and the simulated virtual environment. Subjects then did a short training session with a few randomly chosen configurations from Table 1. For each such configuration subjects were asked to grasp the end of the handle via Phantom and penetrate the tissue layers five times. Subjects were asked to stop as soon as possible once they felt the third layer was penetrated. Subjects continued to practice until they felt confident and comfortable performing the task. The formal trials then began.

Each subject experimented with the all 36 value combinations (trials) in Table 1 in random order. In each trial, subjects performed the same task as in the training session, namely five penetrations of the third tissue layer. Each time the third layer was penetrated, the overshoot was calculated by the program and saved into a file for later analysis. Subjects were encouraged to make comments during the trials. Subjects were also allowed short breaks whenever they claimed fatigue. Each subject took about 1 hour.

\subsection{Participants}

Four students at Simon Fraser University participated in this informal user study. One was from the School of Kinesiology and the other three were from the School of Engineering Science. All participants were right handed.

\subsection{Results and Discussion}

We averaged over the five overshoots for each trial and then over the four subjects, to minimize variation within trial and between subjects. The results are summarized in Table 2 .

If we mark the extreme cases (overshoot greater than $15 \mathrm{~mm}$ is boxed and overshoot less than $5 \mathrm{~mm}$ is shaded), we find almost all the marked cells correspond to $h=80.0$. This indicates that assigning $h$ a large value can result in the best or the worst results in terms of minimizing overshoot, when combined with other factors. The combination of $h=80.0$ and $\Phi=\pi / 6$ is particularly undesirable due to the larger overshoots. The second and third four-bar linkage configurations seem to be preferable over the first, from the distribution of the shaded cells.

Under $h=0.0, \Phi=\pi / 2$ seems to be the worst choice among the three angles we experimented. This is in contrast with the combinations with $h=80.0$, where $\Phi=$ $\pi / 6$ is the worst case. Therefore, the effect of the angles interacts with that of relative position of the handle on the third link.

If we compare the numbers under $l_{h}=140$ with those under $l_{h}=180$, in 14 pairs out of 18 a smaller overshoot is observed for $l_{h}=180$, which may suggest that $l_{h}=180$ is preferable over $l_{h}=140$.

A complete analysis of the effect of the four variables $\left(h, \Phi, l_{h}\right.$ and four-bar configuration) that interact with one another would be difficult to interpret and hard to generalize to general complex mechanisms. However, a table like Table 2 is always easy to construct with a user study. By observation, we can pick out the better combinations of variables based on average overshoot 
With visual feedback alone, typical in traditional CAD, our overshoot data would not be as meaningful as what we obtained because the operator's performance depends on the force characteristics of the mechanism.

Table 2: User study results. Table entry is overshoot in millimeters, with standard deviation in parentheses. Angles are in radians. Configurations 1, 2 and 3 are illustrated in Figure 9. $\Phi, h$, and $I_{h}$ have the same meaning as in Figure 9. Overshoot greater than $15 \mathrm{~mm}$ are put into rectangles; those less than or equal to $5 \mathrm{~mm}$ are shaded gray.

\begin{tabular}{|c|c|c|c|c|c|}
\hline & \multicolumn{2}{|c|}{$h=0.0$} & \multicolumn{2}{|c|}{$h=80.0$} \\
\hline & & $\begin{array}{l}l_{h}= \\
140\end{array}$ & $\begin{array}{c}l_{h}= \\
180\end{array}$ & $\begin{array}{l}l_{h}= \\
140\end{array}$ & $\begin{array}{c}l_{h}= \\
180\end{array}$ \\
\hline \multirow{3}{*}{$\begin{array}{c}\text { Four bar } \\
1\end{array}$} & $\Phi=0$ & $\begin{array}{c}7.9 \\
(6.2) \\
\end{array}$ & $\begin{array}{c}9.5 \\
(9.4) \\
\end{array}$ & $\begin{array}{c}7.7 \\
(9.6) \\
\end{array}$ & $\begin{array}{c}11.4 \\
(14.9) \\
\end{array}$ \\
\hline & $\Phi=\pi / 2$ & $\begin{array}{c}13.4 \\
(10.8)\end{array}$ & $\begin{array}{l}11.9 \\
(9.0)\end{array}$ & $\begin{array}{l}11.2 \\
(7.6)\end{array}$ & $\begin{array}{l}10.3 \\
(9.5)\end{array}$ \\
\hline & $\Phi=5 \pi / 6$ & $\begin{array}{c}13.2 \\
(11.0)\end{array}$ & $\begin{array}{c}8.0 \\
(5.3)\end{array}$ & \begin{tabular}{|l|}
31.0 \\
$(21.1)$
\end{tabular} & \begin{tabular}{|l|}
21.8 \\
$(18.2)$
\end{tabular} \\
\hline \multirow{3}{*}{$\begin{array}{c}\text { Four bar } \\
2\end{array}$} & $\Phi=0$ & $\begin{array}{c}9.2 \\
(9.0) \\
\end{array}$ & $\begin{array}{c}8.3 \\
(11.7) \\
\end{array}$ & $\begin{array}{c}4.1 \\
(1.6) \\
\end{array}$ & $\begin{array}{c}7.3 \\
(8.0) \\
\end{array}$ \\
\hline & $\Phi=\pi / 2$ & $\begin{array}{c}10.4 \\
(11.0) \\
\end{array}$ & $\begin{array}{l}10.0 \\
(9.7) \\
\end{array}$ & $\begin{array}{c}7.3 \\
(4.9) \\
\end{array}$ & $\begin{array}{c}3.9 \\
(2.1) \\
\end{array}$ \\
\hline & $\Phi=5 \pi / 6$ & $\begin{array}{c}7.7 \\
(6.3) \\
\end{array}$ & $\begin{array}{c}4.6 \\
(3.2)\end{array}$ & \begin{tabular}{|l|}
15.2 \\
$(7.0)$ \\
\end{tabular} & \begin{tabular}{|l|}
17.8 \\
$(14.6)$
\end{tabular} \\
\hline \multirow{3}{*}{$\begin{array}{c}\text { Four bar } \\
3\end{array}$} & $\Phi=0$ & $\begin{array}{c}9.1 \\
(7.4)\end{array}$ & $\begin{array}{c}5.0 \\
(3.3)\end{array}$ & $\begin{array}{c}4.9 \\
(2.4)\end{array}$ & $\begin{array}{c}4.7 \\
(2.8)\end{array}$ \\
\hline & $\Phi=\pi / 2$ & $\begin{array}{c}12.2 \\
(15.1)\end{array}$ & $\begin{array}{c}7.6 \\
(6.8)\end{array}$ & $\begin{array}{c}7.1 \\
(7.6)\end{array}$ & $\begin{array}{c}4.8 \\
(4.1)\end{array}$ \\
\hline & $\Phi=5 \pi / 6$ & $\begin{array}{c}6.5 \\
(3.4)\end{array}$ & $\begin{array}{c}6.4 \\
(3.6)\end{array}$ & $\frac{20.0}{(12.6)}$ & $\begin{array}{l}14.5 \\
(3.2)\end{array}$ \\
\hline
\end{tabular}

Haptic feedback also seems to lead to intuitive understanding of the mechanism in our preliminary user study. During the experiment all subjects commented that it is more difficult to manipulate the mechanism when $\Phi$ $=5 \pi / 6$. One subject observed that when $h=80.0$ and $l_{h}=$ 180.0 , the lever arm is so large that the force change due to penetration of tissue layers becomes indistinct, which cannot be predicted by beforehand calculations. These observations are only possible when the mechanism is simulated haptically.

It can be proposed that the case study of an amniocentesis mechanism simulation indicates that haptic rendering can assist in designing mechanisms whose force characteristics are essential to the performance of human operators. Intended users can try out such mechanisms as if in the real application context, make intuitive evaluations of the mechanism, and identify better designs.

\subsection{Conclusions and discussion.}

This paper described a preliminary study of Haptic Aided Design (HAD) in the form of an amniocentesis mechanism design. The mechanism was modeled as a four-bar linkage that can be manipulated to penetrate a three-layer tissue model with different force properties at each layer. A collision detection algorithm was implemented to detect the contact the needle makes with the tissue model. To test the effectiveness of haptic feedback in the mechanism design process, we conducted an informal user study. The results suggest that haptic feedback assists in distinguishing good and bad designs.

This research can be further extended in several directions. First, the skin and amniotic sac layers of our tissue model do not have thickness because they are surface meshes. In future studies these layers may be extended to layers with different thickness using volumetric representations. Second, the force properties of the layers in our tissue model may be tuned to match the force profiles obtained from real tissues, such as that described in [8]. Third, the collision detection algorithm in this study can be refined to handle extravagant cases. Fourth, the concept of Haptic Aided Design (HAD) was examined in a very narrow scope in this study: an amniocentesis mechanism design. The same concept could be applied to more general designs where humans are the users and evaluators, e.g. toys. The HAD applications in these different domains require further investigation.

\section{References}

[1] Hollerbach, J.M., E. Cohen, W. Thompson, R. Freier, D. Johnson, A. Nahvi, D. Nelson, and T.V.Thomson II, "Haptic Interfacing for Virtual Prototyping of Mechanical CAD Designs", Proceedings of 1997 ASME Desgin Engineering Technical Conferences, Sept 14-17, 1997, Sacramento, California, USA.

[2] Nahvi, A., D.D. Nelson, J.M. Hollerbach, and D.E. Johnson, "Haptic Manipulation of Mechanisms from Mechanical CAD Designs", Proceedings of IEEE Intl. Conf. Robotics and Automation, Leuven, Belgium, May 16-21, 1998, p375-380.

[3] Maekawa, H. and J.M. Hollerbach, "Haptic Display for Object Graspign and Manipulating in Virtual Environment", Proceedings of the 1998 IEEE International Conference on Robotics \& Automation, Leuven, Belgium, May 1998, pp25662573.

[4] Hollerbach, J.M., E. Cohen, W.B. Thompson, R. Freier, D.E. Johnson, and T.V. Thompson II, "Virtual Prototyping for Human-Centric Design", Proceedings of 2000 NSF Design and Manufacturing Research Conf., Vancouver, Canada, Jan 3-6, 2000.

[5] Viorel G.P. and G.C. Burdea, "Dextrous Haptic Interface for JACK ${ }^{\mathrm{TM}}$ ", Proceedings of the ASME Dynamic Systems and Control Division, DSC-Vol.64, 1998, pp.189-194.

[6] Jayaram, S., U. Jayaram, Y. Wang, H. Tirumali, K. Lyons, and P.F. Hart, "VADE: A Virtual Assembly Design 
Environment", IEEE Computer Graphics and Its Applications, November 1999, pp44-50.

[7] Zhang, J., Payandeh, S. and Dill, J.,"Haptic subdivision: an Approach to Defining Levels of Detail in Haptic Rendering", Proceedings of the $10^{\text {th }}$ Symposium on Haptic Interfaces for Virtual Environment and Teleoperator Systems, Orlando, FL, March, 2002, pp201-208.

[8] Holton, L. L. H., "Force Models for Needle Insertion Created from Measured Needle Puncture Data", Proceedings of Medicine Meets Virtual Reality, 2001, pp. 180-186.

[9] Gorman, P., T. Krummel, R. Webster, M. Smith, D. Hutchens, "A Prototype Haptic Lumbar Puncture Simulator", Medicine Meets Virtual Reality 2000, IOS Press, 2000, pp.106109.

[10] Dang, T., M. A. Srinivasan, and T. M. Annaswamy, "Development and Evaluation of an Epidural Injection Simulator with Force Feedback for Medical Training", Proceedings of Medicine Meets Virtual Reality, 2001, pp. 97102.

[11] Meseure, P. and C. Chaillou, "Deformable Body Simulation with Adaptive Subdivision and Cuttings", Proceedings of the WSCG'97 Conference, Plzen, February 1014, 1997, pp.361-370.

[12] Hutchinson, D., M. Preston and T. Hewitt, "Adaptive Refinement for Mass/Spring Simulations", $7^{\text {th }}$ Eurographics Worskshop on Animation \& Simulation, Poitiers, August 31September 1, 1996, pp 31-45.

[13] Zhang, D. and M.M.F. Yuen, "Cloth Simulation using Multilevel Meshes", Computers \& Graphics, 25 (2001), pp.383389.

[14] Dehunne, G., M. Desbrun, M. Cani and A. H. Barr, "Dynamic Real-time Deformations using Space \& time Adaptive Sampling”, SIGGRAPH 2001, 12-17 August 2001, Los Angeles, USA.

[15] Johnson, D. E., E. Cohen, "An Improved Method for Haptic Tracing of a Sculptured Surface", Proceedings of the ASME Dynamic Systems and Control Division, DSC-Vol.64, 1998, pp 243-248.

[16] Gottschalk, S., M. C. Lin and D. Manocha, "OBBTree: A Hierarchical Structure for Rapid Interference Detection", SIGGRPAPH 1996, 4-9 August 1996, New Orleans, Louisiana, USA.

\section{Appendix}

We note that if the mesh is significantly concave, like the 1D mesh (connected line segments) shown below, the algorithm FindClosestVertex might not work. This situation is illustrated by the 1D mesh in Figure 10. The filled square is the probe tip. We want to detect collisions between the square and the mesh. At time $\mathrm{t}=\mathrm{t}_{0}$ vertex $\mathrm{D}$ on the mesh is the closest point. At time $t=t_{1}$, the square has moved close to vertex A. If at time $\mathrm{t}=\mathrm{t}_{1}$ we run algorithm FindClosestVertex, because no neighbor vertex $(\mathrm{C}, \mathrm{E})$ is closer to the square than $\mathrm{D}$, no new closest vertex will be found, which causes the algorithm to fail.
Fortunately, the tissue model we used does not have such extreme concave features, and a user's normal movement between two consecutive model updates is small compared to the length of the polygon edges of the meshes, so our algorithm worked well during the user study.

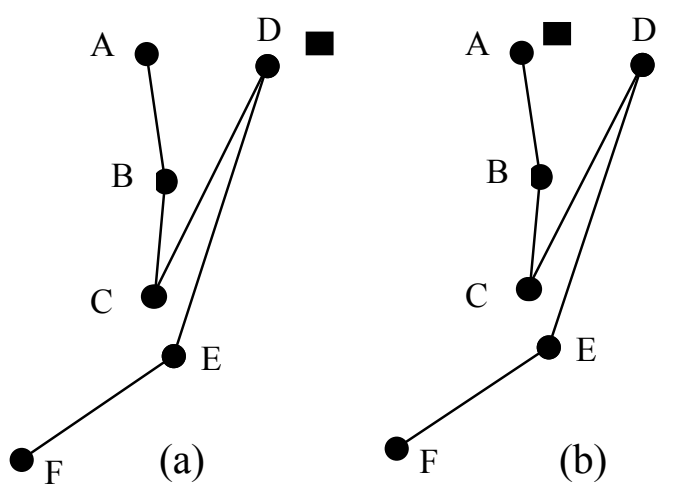

Figure 10: A case where our collision detection method does not work. (a) $t=t_{0}$. (b) $t=t_{1}$. The closest point of the mesh to the end of probe was $D$ at $t=t_{0}$ and is $A$ at $t=t_{1}$, but algorithm FindClosestVertex still returns an empty pointer, signaling no other vertex is closer to the end of probe than D. 